\section{Universal screening of Lynch syndrome is ready for implementation}

\section{To the Editor}

A key determinant in facilitating the implementation of a screening program is a firm commitment from decisionmakers to allocate the necessary resources. With this in mind, we considered it valuable to summarize the results of existing economic evaluations of Lynch syndrome (LS) screening programs so that we may provide decision-makers with an extensive overview of the programs likely to be cost-effective. The results of our systematic approach confirmed that "from a health-care perspective, the cost-effectiveness of both universal and age-targeted CRC-based LS screening is acceptable in terms of willingness-to-pay for health gains". ${ }^{1}$ We were not merely interested in a comparison of incremental cost-effectiveness ratios (ICERs), but also felt it important to carry out a comprehensive classification of LS screening programs considered to be cost-effective in the peer-reviewed studies that analyzed them. Because ICER estimates extrapolated from the context (e.g., assumptions, model parameters, setting, etc.) are not informative, we decided to assess the cost-effectiveness results within each study. Clearly, as Dr Grosse says, ${ }^{2}$ context matters, but for this very reason we refrained from adjusting ICER estimates for inflation as this, by itself, would be insufficient to make very different studies comparable. Instead, our approach allows readers easy access to ready-to-use information, eliminating both the burden and risk of erroneous interpretations.

Furthermore, because context is also crucial for any future implementation of screening programs, we pointed out in our review that "both the design of the screening program and the implementation process will need to be tailored to the characteristics of target populations and health-care systems to ensure the translation of cost-effectiveness evidence into the real-world". Therefore, while we agree with Grosse about the importance of context and thank him for the additional data provided, ${ }^{2}$ we do not believe this changes the conclusions of our study, for the reasons reported below.

The final results from Mvundura et al. ${ }^{3}$ (reference 30 in our review) showed that universal screening for LS using immunohistochemistry and BRAF testing as preliminary tests costs less than $\$ 40,000$ per life year gained (LYG) compared with age-targeted screening (age $<50$ years). Accordingly, the authors concluded that LS screening of all newly diagnosed colorectal cancer (CRC) patients is cost-effective. After revising the model, the authors concluded that a "universal offer of testing for LS in newly diagnosed patients with CRC in the United States still appears cost-effective", and further that the "revised ICER is still low relative to usual costeffectiveness thresholds of $\$ 50,000-\$ 100,000$ per LYG". Furthermore, an ICER of $\$ 52,211$ per LYG (or about $\$ 63,000$ per quality-adjusted life year (QALY)), as calculated by Grosse, ${ }^{2}$ for universal versus age-targeted screening is still below the advocated willingness-to-pay threshold of $\$ 100,000$ per QALY or LYG. ${ }^{4}$

In the base-case analysis of the Canadian Agency for Drugs and Technologies in Health study ${ }^{5}$ (reference 43 in our review), the ICER derived from the comparison between a universal screening program, based on immunohistochemistry testing plus MLH1 hypermethylation testing, and an agetargeted CRC-based program (age $<70$ years) was $\$ 28,902$ per QALY. This estimate proved less favorable when subjected to a sensitivity analysis in which alternative costs were applied to the diagnostic tests. Thus, for the base-case analysis, the costs of diagnostic tests at a public hospital laboratory were used, while the sensitivity analysis used costs from a private laboratory, suggesting that the result for actual costeffectiveness would be somewhere between the two values. Hence, health-care organizations will need to find the right balance of public and private laboratory involvement to ensure the successful implementation of universal LS screening. However, as the authors of the Canadian Agency for Drugs and Technologies in Health study argued, the costs of germline testing are expected to decline in the coming years and this should improve the overall cost-effectiveness of LS screening.

We did not report the ICER estimate for universal versus age-targeted screening (age $<70$ years) from Ladabaum et al. ${ }^{6}$ (reference 33 in our review) because we included only the estimates from the base-case analyses of the studies surveyed. However, if we had included this estimate, our conclusions would not have changed. In their analysis, the ICER estimate for universal versus age-targeted screening was $\$ 88,700$ per LYG, which is below the acceptable willingness-to-pay threshold of $\$ 100,000$. $^{4}$

In conclusion, universal screening is good value for money and, together with near-universal screening (age-targeted screening for individuals $<70$ years), it should be proposed to decision-makers as a potential LS screening program. We agree with Grosse that context matters, and this is why each individual health system should select the most suitable model and adapt it for its own requirements. The challenges of LS screening will be to reach all CRC patients affected by LS, inform and test as many relatives as possible, offer effective surveillance interventions to reduce cancer morbidity and mortality among mutation carriers, and thus effectively maximize the health status of the population with the available resources. 


\section{ACKNOWLEDGEMENTS}

This work is supported by the Personalized Prevention of Chronic Diseases Consortium project, funded by the European Union's Horizon 2020 research and innovation program Marie Skłodowska-Curie Research and Innovation Staff Exchange, under grant agreement 645740 .

\section{DISCLOSURE}

The authors declare no conflicts of interest.

Marco Di Marco, $M D^{1}$, Elvira D’Andrea, $M D, M P H^{1,2}$ and Paolo Villari, $\mathrm{MD}, \mathrm{MPH}^{1}$

${ }^{1}$ Department of Public Health and Infectious Diseases, Sapienza University of Rome, Rome, Italy; ${ }^{2}$ Division of Pharmacoepidemiology and Pharmacoeconomics, Brigham and Women's Hospital/Harvard Medical School, Boston, MA, USA. Correspondence: Marco Di Marco (marcodimarco@live.it)

\section{REFERENCES}

1. Di Marco M, D'Andrea $E$, Panic N, et al. Which Lynch syndrome screening programs could be implemented in the "real world"? A systematic review of economic evaluations. Genet Med. 2018. https://doi.org/ 10.1038/gim.2017.244.

2. Grosse S. Is universal tumor testing for Lynch syndrome cost-effective? It depends! Genet Med. 2018 (this issue).

3. Mvundura M, Grosse SD, Hampel H, Palomaki GE. The cost-effectiveness of genetic testing strategies for Lynch syndrome among newly diagnosed patients with colorectal cancer. Genet Med. 2010;12:93-104.

4. Neumann PJ, Cohen JT, Weinstein MC. Updating cost-effectiveness-the curious resilience of the $\$ 50,000$-per-QALY threshold. N Engl J Med. 2014;371:796-7.

5. Canadian Agency for Drugs, Technologies in HealthDNA Mismatch Repair Deficiency Tumour Testing for Patients with Colorectal Cancer: A Health Technology Assessment. CADTH Optimal Use Report, vol. 5, no. 3b). Ottawa, Canada, 2016.

6. Ladabaum U, Wang G, Terdiman J et al. Strategies to identify the Lynch syndrome among patients with colorectal cancer: a cost-effectiveness analysis. Ann Intern Med. 2011;155:69-79.

Advance online publication $\mathrm{xx}$ xx 2018. doi:10.1038/s41436-018-0027-3 